\title{
BioéthiqueOnline
}

\section{Trouble du spectre de l'autisme : une agentivité morale objective, rigoriste et émotionnelle}

\section{Sarah Arnaud}

Volume 5, 2016

URI : https://id.erudit.org/iderudit/1044270ar

DOI : https://doi.org/10.7202/1044270ar

Aller au sommaire du numéro

Éditeur(s)

BioéthiqueOnline

ISSN

1923-2799 (numérique)

Découvrir la revue

\section{Citer cet article}

Arnaud, S. (2016). Trouble du spectre de l'autisme : une agentivité morale objective, rigoriste et émotionnelle. BioéthiqueOnline, 5.

https://doi.org/10.7202/1044270ar

\section{Résumé de l'article}

Le trouble du spectre de l'autisme (TSA) désigne un ensemble de troubles neurodéveloppementaux caractérisé par des difficultés de communication et d'interactions sociales, ainsi que des comportements, intérêts et activités restreints et répétitifs (1). Concernant les interactions sociales, les personnes autistes auraient notamment des difficultés d'empathie, souvent considérées comme altérant leurs capacités morales. Ainsi, plusieurs études en psychologie et en neuroscience tentent de détecter des processus empathiques défaillants à l'origine d'altérations de leur compréhension de la moralité (2-4). Les problèmes sociaux des personnes autistes se manifestent aussi par des difficultés dans leur vie affective qui concernent leurs propres états émotionnels (5-9). Je suggère que les particularités morales des personnes autistes sont issues de leurs particularités d'accès émotionnel, soit la façon dont elles se rapportent à leurs propres émotions, et non pas de déficits d'empathie. Je montrerai que ces particularités ne les empêchent en aucun cas de faire partie de la communauté morale. Les personnes autistes peuvent être de rigoureux agents moraux, c'est-à-dire qu'elles présentent une certaine intransigeance et inflexibilité morale. Ces particularités seront expliquées. À la lumière des théories sur les processus duaux, je montrerai comment leurs particularités socio-émotionnelles et morales sont toutes deux sous-tendues par une surutilisation de processus de type 2, soit une surutilisation de raisonnements cognitifs délibérés et basés sur des règles, plutôt que des processus automatiques, de type 1 . L'objectif principal de cette étude sera donc de mettre en évidence les particularités qui permettent aux personnes autistes d'être des agents moraux certes inflexibles, mais néanmoins émotionnellement investis dans les situations à caractère moral.
Ce document est protégé par la loi sur le droit d'auteur. L'utilisation des services d’Érudit (y compris la reproduction) est assujettie à sa politique d'utilisation que vous pouvez consulter en ligne.

https://apropos.erudit.org/fr/usagers/politique-dutilisation/ 


\title{
Trouble du spectre de l'autisme : Une agentivité morale objective, rigoriste et émotionnelle
}

\author{
ARTICLE (RÉVISION PAR LES PAIRS / PEER-REVIEWED) \\ Sarah Arnaud ${ }^{1,2}$

Reçu/Received: 12 Aug $2015 \quad$ Publié/Published: 26 May 2016
Éditeurs/Editors: Charles Dupras, Vanessa Chenel \& Jean Poupart
Évaluateurs externes/Peer-Reviewers: Karine Morasse \& Anonymous

2016 S Arnaud, Creative Commons Attribution 4.0 International License

\section{Résumé}

Le trouble du spectre de l'autisme (TSA) désigne un ensemble de troubles neurodéveloppementaux caractérisé par des difficultés de communication et d'interactions sociales, ainsi que des comportements, intérêts et activités restreints et répétitifs (1). Concernant les interactions sociales, les personnes autistes auraient notamment des difficultés d'empathie, souvent considérées comme altérant leurs capacités morales. Ainsi, plusieurs études en psychologie et en neuroscience tentent de détecter des processus empathiques défaillants à l'origine d'altérations de leur compréhension de la moralité (2-4). Les problèmes sociaux des personnes autistes se manifestent aussi par des difficultés dans leur vie affective qui concernent leurs propres états émotionnels (5-9). Je suggère que les particularités morales des personnes autistes sont issues de leurs particularités d'accès émotionnel, soit la façon dont elles se rapportent à leurs propres émotions, et non pas de déficits d'empathie. Je montrerai que ces particularités ne les empêchent en aucun cas de faire partie de la communauté morale. Les personnes autistes peuvent être de rigoureux agents moraux, c'est-à-dire qu'elles présentent une certaine intransigeance et inflexibilité morale. Ces particularités seront expliquées. À la lumière des théories sur les processus duaux, je montrerai comment leurs particularités socio-émotionnelles et morales sont toutes deux sous-tendues par une surutilisation de processus de type 2 , soit une surutilisation de raisonnements cognitifs délibérés et basés sur des règles, plutôt que des processus automatiques, de type 1 . L'objectif principal de cette étude sera donc de mettre en évidence les particularités qui permettent aux personnes autistes d'être des agents moraux certes inflexibles, mais néanmoins émotionnellement investis dans les situations à caractère moral.

\section{Mots clés}

TSA, autisme, moralité, accès émotionnel, émotions, empathie

\section{Abstract}

Autism Spectrum Disorder (ASD) refers to a set of neurodevelopmental disorders characterized by deficits in social communication and social interactions, as well as restricted and repetitive patterns of behaviour, interests and activities (1). Social interaction deficits notably include difficulties in empathic processes, often considered as affecting moral abilities in people suffering from ASD. Several studies in psychology and neuroscience have attempted to detect disturbed empathic processes that cause alterations in their understanding of morality (2-4). Social problems in autistic people are manifested by difficulties in their affective lives concerning their own emotional states (5-9). In this paper, I suggest that moral particularities of people with autism are a consequence of their particularities in emotional access, namely the way they relate to their own emotions, and not a consequence of empathic deficits. I will show that these particularities do not prevent them from belonging to the moral community. People with autism can indeed be rigorous moral agents, that is to say, they display a tendency to moral intransigence and inflexibility. These characteristics will be explained. In light of dual process theories, I will show how socio-emotional and moral particularities are underpinned by an overuse of type 2 processes, namely an overuse of deliberate and rule-based cognitive reasoning, instead of type 1 automatic processes. The main purpose of this study is to highlight the particularities that allow people with autism to be moral agents who, although inflexible, are nonetheless emotionally committed in moral situations.

\section{Keywords}

ASD, autism, morality, emotional access, emotions, empathy
Responsabilités des évaluateurs externes Les évaluations des examinateurs externes sont prises en considération de façon sérieuse par les éditeurs et les auteurs dans la préparation des manuscrits pour publication. Toutefois, être nommé comme examinateur n'indique pas nécessairement l'approbation de ce manuscrit. Les éditeurs de BioéthiqueOnline assument la responsabilité entière de l'acceptation finale et la publication d'un article.

\section{Peer-reviewer responsibilities}

Reviewer evaluations are given serious consideration by the editors and authors in the preparation of manuscripts for publication. Nonetheless, being named as a reviewer does not necessarily denote approval of a manuscript; the editors of BioéthiqueOnline take full responsibility for final acceptance and publication of an article 
Affiliations des auteurs / Author Affiliations

${ }^{1}$ Département de philosophie, Université du Québec à Montréal, Montréal, Canada

${ }^{2}$ Programme de philosophie, "Sciences normes décision », Université Paris-Sorbonne, Paris, France

\section{Correspondance / Correspondence}

Sarah Arnaud, sarah.audrey.arnaud@gmail.com

\section{Remerciements}

Je tiens à remercier les organisatrices et organisateurs de la conférence en Neuroéthique, l'éditeur de cet article Jean Poupart de BioéthiqueOnline, les évaluateurs externes, ainsi que Eamon Leonard et Luc Faucher, pour leurs commentaires et suggestions sur les versions antérieures de ce texte.

\section{Conflit d'intérêts}

Aucun déclaré

\section{Acknowledgements}

I would like to thank the organizers of the Neuroethics conference, the BioéthiqueOnline editor of this article Jean Poupart, the external peer-reviewers, as well as Eamon Leonard and Luc Faucher, for their comments and suggestions on previous versions of this text.

Conflicts of Interest

None to declare

\section{Introduction}

Chez les neurotypiques, le fait de ressentir certaines émotions par rapport à autrui est considéré comme permettant le développement de capacités morales. Un enfant qui se sent coupable d'avoir blessé un camarade va commencer à comprendre qu'il est moralement condamnable de faire du mal à autrui. À l'âge adulte, les émotions semblent également impliquées dans notre moralité : le dégoût favorise la condamnation de l'inceste, la colère permet de reconnaitre une offense, etc.... Les personnes dites "psychopathes " sont caractérisées par un déficit important d'empathie. Ainsi, ne percevant pas le mal fait à autrui, et étant qualifiés comme exemptes de culpabilité et de remords, certains pensent que leur problème avec la moralité est dû à leur manque d'empathie $(10,11)$.

La recherche dans le domaine de l'autisme met en évidence de nombreux déficits ou particularités en ce qui concerne les processus émotionnels des personnes autistes. Les difficultés d'interactions sociales sont une des caractéristiques principales des troubles du spectre de l'autisme (TSA). Elles se manifestent notamment par des particularités émotionnelles, désignées par la $5^{\mathrm{e}}$ version du Diagnostic and Statistical Manual of Mental Disorders (DSM-5) comme un déficit de "réciprocité socio-émotionnelle »(1), qui peuvent atteindre différents degrés de sévérité, l'autisme étant une condition particulièrement hétérogène ${ }^{1}$. Si toutes les personnes autistes présentent des particularités dans leur façon d'interagir avec autrui, certaines parviendront à utiliser des stratégies pour compenser leurs difficultés d'interactions sociales alors que d'autres souffriront plus nettement de ce manque d'interaction. Ces difficultés relationnelles peuvent donc se manifester de différentes manières d'une personne à l'autre, mais elles ont souvent été mises sur le compte d'incapacités à comprendre la perspective d'autrui (12) et de certaines difficultés d'empathie $(5,13,14)$ confirmées par de récentes études en neuroscience qui montrent des altérations de fonctionnement des neurones miroirs chez ce groupe de sujets $(15,16)$. De la même manière que la moralité des personnes psychopathes est possiblement en lien avec leurs déficits d'empathie, la possibilité pour les personnes autistes de juger un acte comme étant condamnable ou louable, d'évaluer des situations à la lumière d'un système de normes, et d'être apte à juger moralement leurs actes ou ceux d'autrui, autrement dit la capacité de développer une agentivité morale, a donc été remise en question (17).

Cependant, si les personnes autistes sont souvent sujettes à une " maladresse morale » (18), elles semblent tout à fait capables, contrairement aux psychopathes, de distinguer les règles morales et conventionnelles, et ce, qu'elles soient capables ou non de comprendre la perspective d'autrui (19). En fait, cette capacité à distinguer entre ces deux types de règles ne semble pas poser de difficulté

\footnotetext{
${ }^{1}$ Les échelles de sévérité incluses dans le DSM-5 pour les différents troubles rendent compte de l'hétérogénéité de l'autisme. La recherche distingue aussi par exemple les personnes autistes de haut niveau, c'est-à-dire n'ayant pas de déficience intellectuelle, des autres personnes autistes.
} 
aux personnes autistes, quel que soit le degré de sévérité de leurs troubles socio-communicationnels. Certaines personnes autistes de haut niveau semblent même parfois construire volontairement, et avec intérêt, une moralité applicable à la vie en société (20). Pourquoi alors les personnes autistes sont-elles encore sujettes à certaines particularités morales et quels sont les liens de ces particularités avec leur vie affective? Ces particularités remettent-elles en question la validité de leurs jugements moraux? Les réponses à ces questions auront des implications éthiques importantes dans la mesure où elles trancheront sur l'inclusion ou l'exclusion des personnes autistes dans la communauté morale. Une étude de l'agentivité morale des personnes autistes devrait déterminer si elles doivent être considérées comme autonomes moralement ou si elles ne sont au contraire pas responsables de leurs jugements et comportements à caractère moral.

Cet article a pour but principal de montrer que les personnes autistes peuvent bel et bien être considérées comme des agents moraux et ainsi appartenir à la communauté morale, c'est-à-dire à l'ensemble des individus à qui on reconnait des droits et des devoirs moraux. II faudra pour cela expliquer les fondements de leurs particularités morales et émotionnelles, c'est-à-dire expliquer la façon dont les personnes autistes raisonnent moralement et la façon dont elles se rapportent à leurs états émotionnels, puis montrer les liens existants entre ces deux processus. Étant donné qu'il existe différents modèles théoriques de la moralité, un objectif nécessaire à l'appui de l'hypothèse principale sera de décrire ces différents modèles et de montrer que l'agentivité morale des personnes autistes est compatible avec chacun d'entre eux. II sera ainsi établi que leur rapport à la moralité se distingue nettement de celui des personnes psychopathes, pour qui la possibilité d'une responsabilité morale est remise en question. L'agentivité morale dans l'autisme sera qualifiée comme étant sujette à une certaine inflexibilité et rigueur, causé par un accès contrôlé et raisonné aux normes morales et aux émotions, mais néanmoins impliquant un investissement émotionnel important.

\section{Une agentivité morale inflexible et rigoriste}

L'agentivité morale joue un rôle primordial dans le développement des individus et leurs rapports sociaux, dans la mesure où elle permet de se comporter moralement, en adéquation avec les normes légales et sociales de la société. L'agentivité morale est conçue comme nécessaire à la responsabilité morale, c'est-à-dire au fait qu'une personne puisse être tenue responsable de ses actes. Les enfants par exemple n'ont pas la même responsabilité morale que les adultes : la loi leur reconnait bien des droits et devoirs, mais ils sont adaptés à leur développement. Ils font partie de la communauté morale au sens large, c'est-à-dire qu'on leur témoigne une considération morale, mais pas en un sens plus étroit ; ce sont souvent leurs parents ou éducateurs qui portent une responsabilité morale en leur nom, leur agentivité morale n'étant pas encore pleinement développée. Leurs décisions sont soumises à l'autorité de leurs tuteurs. Si les personnes autistes ne pouvaient pas développer d'agentivité morale, leurs droits et devoirs pourraient aussi s'avérer différents de ceux des adultes neurotypiques, et comparables à ceux des enfants par exemple. Leur place dans la communauté morale en un sens étroit serait alors remise en question.

La façon dont l'agentivité morale se développe est expliquée différemment par différentes conceptions de la moralité. Selon les modèles rationalistes de la moralité, le comportement moral interagit avec le raisonnement moral : la saisie des réalités morales est issue de la réflexion ou de la connaissance des règles morales. Ces théories ${ }^{2}$, contrairement aux modèles sentimentalistes de la moralité, soutiennent que les sentiments moraux, ou les émotions associées à la moralité, sont tout au plus une conséquence de notre raisonnement moral. Ils ne sont en aucun cas nécessaires au développement d'une moralité (10). Une personne qui reconnait les transgressions morales et obéit

\footnotetext{
${ }^{2}$ Souvent associées aux idées de Kant, qui théorise la moralité par une certaine neutralité, rendue possible par l'usage de la raison ; et dont s'inspirera plus tard le psychologue Kohlberg pour qui cette perspective neutre de la moralité correspond à un stade avancé du développement moral des individus, les deux théoriciens accordant donc une place importante à l'usage de la raison dans la moralité.
} 
aux lois et règles morales en se détachant de toute dimension affective sera alors un-e agent-e moral-e selon les modèles rationalistes $(21,22)$.

L'apprentissage des règles par les personnes autistes implique une connaissance qui permet d'agir moralement. Contrairement aux personnes psychopathes, elles peuvent faire la distinction entre les règles morales (ex. : l'interdiction de tuer) et les règles conventionnelles (ex. : ne pas mâcher de la gomme en classe)(23), et ainsi distinguer les transgressions morales des transgressions conventionnelles (4). Les personnes autistes de haut niveau vivent même dans " un monde social très normatif » (24). En effet, ces dernières " peuvent développer ou découvrir des règles morales et des principes de conduites pour [elles]-mêmes grâce au raisonnement " (10). Leurs difficultés à extraire des règles sociales abstraites à partir de leurs expériences personnelles peuvent être compensées par des efforts de catégorisation et un renforcement des aspects concrets de ces règles (25). Le cas de Temple Grandin montre que cette catégorisation peut se faire par soi-même chez certaines personnes autistes de haut niveau. En effet, elle a elle-même développé un système complexe pour comprendre la morale et appliquer les règles de conduite. II s'agit selon elle d'un « programme de prise de décision pour savoir si les règles [peuvent] être enfreintes en classifiant le mal commis en trois catégories " (20, traduction libre). Ainsi, grâce à ce " système de classification ", elle peut appliquer des règles de conduite. Temple Grandin adopte alors un comportement moral qu'elle a construit grâce au raisonnement sur ce qui cause du tort à autrui en fonction des sanctions potentielles encourues. Les personnes autistes semblent donc pouvoir être qualifiées d'agents moraux, dans la mesure où elles distinguent ce qui est normatif de ce qui est conventionnel et détectent sans difficulté les transgressions des règles morales. Leur comportement est donc en adéquation avec la moralité telle qu'elle est conçue par les modèles rationalistes. Certaines personnes autistes de haut niveau vont même pouvoir développer une théorie morale par ellesmêmes, comme le montre le cas de Grandin.

La moralité dans les TSA peut par ailleurs s'avérer exemplaire, et supérieure à celle des neurotypiques si l'on suit les modèles rationalistes. En effet, les personnes autistes vont se montrer inflexibles devant certaines situations. La fameuse réaction du jeune homme autiste à qui l'on raconte I'histoire d'une femme sans ressource qui vole dans un supermarché l'illustre bien : "Tout le monde doit passer à la caisse. C'est illégal de ne pas passer à la caisse. Elle devrait être arrêtée » (traduction libre), affirme le jeune homme autiste (26). II condamnerait l'acte malgré des circonstances qui auront tendance à influer sur le jugement de la plupart des neurotypiques, comme le fait que cette femme soit sans argent et doive nourrir ses enfants. L'étude de Bolling et collègues montre bien cette capacité accrue à condamner un acte moralement. En effet, ils rapportent une hyperactivation de l'insula droite et du cortex préfrontal chez des enfants autistes pendant l'observation de la violation d'une règle, ce qui suggère une détection plus importante des transgressions morales que chez les neurotypiques comparé-e-s (27).

Ainsi, les personnes autistes observeraient plus fortement les torts causés à autrui comme des transgressions, et ce, quelles que soient les circonstances (qui viennent chez les neurotypiques atténuer la désapprobation de la situation). Selon les modèles rationalistes de la moralité, les personnes autistes sont donc d'excellents agents moraux. Les transgressions morales étant particulièrement bien détectées, leurs jugements moraux ne faillissent pas. Leur apitoiement devant une situation par exemple, ne les mène pas vers un jugement moral moins sévère, autrement dit, leurs émotions ne les détournent pas de leur jugement moral.

Mais pourquoi la situation difficile de cette femme au supermarché ne mène-t-elle pas ce jeune homme à relativiser sa condamnation? Pourquoi les personnes autistes appliquent les règles morales de façon « machinale » sans considérer les différentes raisons qui auraient pu motiver une personne à enfreindre une règle (28)? Kennett comprend la moralité des personnes autistes comme une morale " kantienne », c'est-à-dire rationaliste, en opposition à une forme " humienne » ou sentimentaliste, qui leur serait inaccessible (10) et qui correspondrait à celle des personnes neurotypiques. Alors que 
les neurotypiques jugeraient la situation moins sévèrement en compatissant avec le malheur de cette femme, les personnes autistes ne ressentiraient pas cet apitoiement. Brewer et collègues défendent une idée similaire, indiquant que les personnes autistes « ne basent pas leurs jugements d'acceptabilité morale sur des informations émotionnelles » (29, traduction libre).

Si cette approche reconnait bien un type de moralité aux personnes autistes, elle les place néanmoins dans une catégorie distincte, qui les exclue du modèle neurotypique ou " normal » de la moralité, leurs émotions n'étant pas constitutives de leurs capacités morales. Si la moralité des personnes autistes semble bien inflexible et donc répond aux principes kantiens, il va être montré que contrairement aux visions de Kennett ou Brewer et collègues, elle est également ressentie et émotionnellement vécue.

\section{Une agentivité morale émotionnellement ressentie}

Admettons qu'une personne connaisse les règles de la moralité grâce à un apprentissage, mais ne ressente aucune émotion associée à la moralité. Elle pourrait par exemple savoir qu'il est interdit de voler ou de tricher, ou qu'il ne faut pas blesser autrui, mais voler ne lui procurerait pas de culpabilité, une personne maltraitée ne lui insufflerait pas de sentiment de pitié. Cette personne serait alors plus à même de tricher si cela peut lui venir en aide, elle viendrait peut-être moins en aide à autrui dans une situation de maltraitance si elle doit prendre le temps de se référer à l'ensemble de ses connaissances en termes de moralité avant de réagir. Elle n'agirait pas moralement si elle n'a aucun intérêt personnel à le faire. Autrement dit, son comportement moral serait moins efficace que celui d'une personne émotionnellement investie dans ses jugements moraux. Une approche purement rationaliste de la moralité ne semble pas prendre en considération cette composante du développement de l'agentivité morale. La moralité des personnes autistes correspond-elle seulement à une approche purement rationaliste? Dans ce cas, leur type d'agentivité morale serait incompatible avec certaines conceptions de la moralité.

Une approche sentimentaliste de la moralité implique justement que le comportement moral soit le produit de nos émotions. La moralité ne dépend pas uniquement de normes objectives préétablies, mais se construit grâce aux émotions que procurent les situations à caractère moral. Ainsi, selon ces approches, l'intuition et l'affect doivent toujours être la cause de l'apparition du raisonnement moral. Par exemple, l'intuitionnisme social de Haidt (30) va impliquer que les notions de bien et de mal soient aussi compréhensibles en termes de sentiments d'approbations ou de désapprobations. Une bonne action est accompagnée d'une émotion à valence positive (la joie, la fierté...) et la condamnation d'un acte dépend d'une émotion de dégoût, de peur, de tristesse, etc. Plus généralement, la majorité des théories morales considère l'importance des émotions dans le développement de nos jugements moraux et montre que les processus moraux et émotionnels sont en interaction.

L'exemple du jeune homme autiste semble alors démontrer un déficit plutôt qu'un avantage en ce qui concerne les capacités morales, car les circonstances particulières qui poussent la femme au vol ne semblent pas lui procurer de sentiment qui influencerait son jugement moral. De plus, Victoria McGeer rappelle que la moralité des personnes autistes est parfois caractérisée par une « rigidité, [et] une insensibilité » (2, traduction libre). Ainsi, on pourrait conclure que les convictions morales des personnes autistes sont simplement issues d'un apprentissage dans lequel leurs émotions n'entrent pas en jeu et que ces convictions ne dénotent donc pas une compréhension des raisons morales. Grandin aurait simplement appris et appliqué des règles pour éviter les sanctions, sans aucun intérêt réel pour les situations à caractère moral. On retrouve dans cette description fictive les caractéristiques d'une personne psychopathe, dénuée d'empathie $(19,31)$ et présentant des déficits 
concernant ses ressentis émotionnels (32), donc étant également dénuée d'intérêt pour la moralité3. En effet, la psychopathie est associée à un manque d'empathie, une indifférence au bien-être ou à la souffrance d'autrui et à des tendances antisociales. La compréhension de la moralité est issue d'un effort cognitif important et non pas d'un sentiment intuitif qui accompagne la situation (11). Ainsi, l'argument de Piers Benn consistait à dire que de la même façon, les difficultés sociales des personnes autistes impliquent une incapacité dans les dimensions morales (17). Cependant, rien pour l'instant ne permet d'affirmer que les personnes autistes ne comprennent pas ou ne ressentent pas les raisons morales qui les poussent à agir. Et leur rapport à la moralité s'éloigne nettement de celui des personnes psychopathes.

Le cas de Grandin illustre bien la volonté d'interagir en société et une motivation à éviter de causer du tort à autrui. Dans son système de classification, elle s'affirme en accord avec certaines règles, qui doivent être respectées pour éviter une des trois catégories du mal que Grandin nomme "les offenses vraiment mauvaises » (20, traduction libre). De plus, elle distingue sa compréhension des règles et les raisons qui les motivent puisque sa troisième catégorie du mal concerne « les actions illégales, mais pas mauvaises » (20, traduction libre). Ainsi, même si les règles et la conduite morale sont apprises - il y a une distinction à connaitre entre ce qui est illégal, considéré comme mal par la société, et ce qui ne l'est pas - les raisons morales peuvent être intuitivement comprises et appréhendées indépendamment de leur valeur " objective » : certaines de ces actions sont illégales à juste titre, donc "vraiment mauvaises », alors que d'autres n'ont pas de bonnes raisons de faire partie de cette catégorie. La moralité dont fait preuve Grandin est donc liée à un certain intérêt, à une compréhension subjective qui va au-delà des règles dictées par le système social, et en cela, elle se distingue considérablement de celle des psychopathes. Blair a montré que les personnes autistes sont sensibles au mal causé à autrui, même lorsque leurs capacités sociales sont très altérées (19). Cela implique une saisie intuitive d'un certain aspect de la moralité, et un ressenti affectif qui l'accompagne, chez les personnes autistes présentant différents niveaux de sévérité au niveau des interactions sociales.

De plus, McGeer montre que les personnes autistes développent une agentivité morale, certes d'une manière différente des neurotypiques, - comme il sera expliqué dans les parties subséquentes de ce texte - mais issue d'une " a-rationnelle passion pour l'ordre » (2, traduction libre), donc d'un rapport affectif fort au besoin d'ordonner le monde, que l'on pourrait comprendre comme un rapport très émotionnel, et surtout qui ne provient pas d'un raisonnement. Un ressenti affectif peut donc accompagner la compréhension de la moralité des personnes autistes, même si ce n'est pas ce ressenti qui entre en jeu dans l'évaluation des situations morales au cas par cas. Ainsi, la construction d'une agentivité morale dans les TSA semble être le produit de leurs intuitions et émotions, ce qui suit une approche sentimentaliste de la moralité.

Ainsi, que l'on se réclame d'un modèle rationaliste ou sentimentaliste de la moralité, les personnes autistes peuvent toujours en principe ${ }^{4}$ être des agents moraux. Aucun critère spécifique à l'autisme n'empêche le développement d'une moralité, et celle-ci comprend des interactions certaines avec des processus émotionnels. Leur moralité n'est donc pas dénuée d'émotion. Pourtant, leur agentivité morale semble différer de celle des neurotypiques.

\footnotetext{
${ }^{3}$ II faut néanmoins noter que la plupart des personnes psychopathes n'ont pas de comportements répréhensibles et ne sont pas hors la loi. Comme l'expliquent Berg et collègues, l'image qui est souvent véhiculée s'éloigne nettement du tableau clinique qui les caractérise (33).

${ }^{4}$ Je précise " en principe », car les déficits cognitifs de nombreuses personnes autistes les empêcheront de développer une agentivité morale. Ces déficits n'étant pas inhérents à la pathologie, mais des caractéristiques comorbides, on peut soutenir que ce ne sont pas les troubles autistiques eux-mêmes qui sont à l'origine de cette impossibilité.
} 


\section{Des évaluations morales systématiques et basées sur des règles}

Bien que tout à fait capables d'agir moralement et de comprendre ce qui sous-tend les normes qu'elles suivent, les personnes autistes sont néanmoins enclines à une certaine " maladresse morale » (18), dans des situations où il faut réagir adéquatement à la détresse d'autrui. Si le cas du manque de flexibilité du jeune homme autiste ne dénote pas d'une absence de moralité, il semble montrer une particularité dans l'application des normes. Le cas de Grandin apparaît également comme idiosyncrasique, car elle doit se référer à son système à chaque fois qu'elle se trouve dans une situation à caractère moral alors que lorsqu'une personne neurotypique formule un jugement moral, elle ne fait pas explicitement référence à un système complet de la moralité ${ }^{5}$. De plus, Kennett et Fine rapportent l'embarras de Jim Sinclair, diagnostiqué autiste, qui se sent impuissant face à la détresse d'une personne qui aurait besoin d'aide. Une détresse qu'il semble pourtant ressentir fortement (18). L'agentivité morale des personnes autistes semble donc particulière en ce qui concerne les situations impliquant une évaluation morale.

Pour comprendre ce qui les sous-tend, De Vignemont et Frith (24) parlent de difficultés à faire le lien entre la détection d'une transgression d'une règle et la détection du sentiment d'autrui, ce qui pourrait expliquer en partie cette maladresse morale. La compréhension du caractère moral de la situation proviendrait d'un apprentissage raisonné de certaines règles et non du sentiment d'autrui qui accompagne la situation. Ce sentiment est pourtant bien éprouvé et, étant lui-même à la source d'un désarroi chez Sinclair, il suggère l'existence d'une certaine forme d'empathie. Ce que disent De Vignemont et Frith est que cette empathie n'éclaire pas la situation morale. La différence d'agentivité avec les neurotypiques semble donc se situer au niveau de l'accès aux règles morales: les personnes autistes élaboreront un jugement moral sur la base du système qu'elles auront construit (comme c'est le cas pour Grandin) et/ou appris, plutôt que sur la base d'un ressenti en ce qui concerne la situation à évaluer. Elles devront se référer à leur raisonnement et au système de moralité adopté pour catégoriser la situation dans le champ de la moralité. Ainsi, devant une situation à caractère moral, leur évaluation sera faite en fonction du système de normes, ce qui sera à l'origine d'une généralisation des situations et de difficultés à relativiser certains évènements en fonction du contexte. Shulman et collègues indiquent à ce sujet que les personnes avec TSA « sont caractérisées par (...) une tendance à sur-généraliser, et une adhérence inflexible aux normes sociales » (traduction libre) lorsqu'il s'agit de justifier leur raisonnement moral (4).

Les neurotypiques au contraire, semblent se référer intuitivement à la moralité, au point de ne pas toujours être en mesure de donner de justification quand ils sont pourtant convaincus qu'une situation est immorale. Haidt donne l'exemple de sujets qui majoritairement condamnent une potentielle relation sexuelle consentie et sans danger entre frères et sœurs. II montre que les sujets qui jugent cet exemple comme étant moralement mauvais ne trouvent pas souvent de raison pour le justifier (30). Cela est dû au fait que la construction de l'évaluation morale soit issue d'une perception intuitive de la situation particulière et pas analysée en fonction de l'ensemble du système moral. Cette perception intuitive permet une évaluation morale rapide des situations et des réactions tout aussi automatiques : une personne neurotypique pourra rassurer et consoler une personne en détresse par exemple. Sans cette automaticité, les agissements s'avèreront plus difficiles, car demandant plus de réflexion (comme en témoigne le désarroi de Sinclair devant la personne en détresse).

Cette différence de fonctionnement dans les TSA est corroborée par les résultats de l'étude de Schneider et collègues sur les corrélats neuronaux du raisonnement moral chez les personnes autistes (3). Cette étude montre une hypoactivation de l'amygdale gauche lors de l'interprétation de dilemmes moraux, ce qui suggère selon les auteurs qu'il n'y a pas d'implication émotionnelle qui mène à des processus de hauts niveaux comme le raisonnement moral ; les dilemmes moraux sont interprétés sans que les émotions ne soient nécessairement engagées. Si l'on suit l'hypothèse de De

\footnotetext{
${ }^{5}$ Sinon la communauté morale serait seulement constituée d'un ensemble de philosophes ou de psychologues moraux et d'autres spécialistes de la moralité!
} 
Vignemont et Frith illustrée par le cas de Sinclair, on peut supposer que l'émotion est engagée avant ou après que le jugement moral soit formulé, mais que ces deux processus sont distincts chez les personnes atteintes d'autisme. Malgré ces différences d'activation cérébrales, les auteurs ne rapportent pas de différences comportementales en ce qui concerne la tâche, ce qui marque bien encore une fois la présence du raisonnement moral. Le fait que les comportements moraux des sujets puissent être sous-tendus par différentes activations cérébrales suggère plutôt que différentes voies sont possibles dans la manière de développer son agentivité morale.

Une manière d'envisager les processus sous-jacents aux particularités qui viennent d'être décrites est de se tourner vers les théories sur les processus duaux. Elles permettront d'expliquer les distinctions entre les personnes autistes et neurotypiques dans la manière de construire une agentivité morale.

\section{Une surutilisation de processus de type 2 dans l'accès aux normes morales}

Développer une agentivité morale implique, entre autres, l'acquisition d'une capacité à évaluer moralement certaines situations. Kennett et Fine ont proposé que la construction des jugements moraux soit issue d'une interaction entre des processus automatiques et d'autres plus contrôlés. Dans une étude récente, Dubljevic et Racine expliquent que les jugements moraux se fondent sur des informations conscientes et apparemment accessibles, mais que ces informations sont en fait soustendues par des processus rapides et inconscients (34). Plus généralement, l'agentivité morale dépendrait à la fois de processus automatiques et de processus de raisonnement conscients (29).

Ces modèles peuvent s'intégrer dans celui des théories des processus duaux, selon lesquelles deux types de processus sont en jeu dans le jugement et la prise de décision. Un ensemble de processus de type 1, intuitifs, automatiques et subjectifs, et un ensemble de processus de type 2, raisonnés, contrôlés et objectifs $(35)^{6}$. Les processus de type 2 vont être utiles pour faire certaines hypothèses de raisonnement ou des simulations mentales. Ils concernent des raisonnements ou des formulations de jugements relativement lents et séquentiels, et sont liés à la mémoire de travail (35). Ce type de raisonnement va par exemple être impliqué dans les jugements moraux concernant certains cas hypothétiques - comme le fait de condamner la femme qui a volé au supermarché parce qu'il est interdit de voler, ou dans les jugements qui font intervenir une information accessible et consciente en général - je jugerai que les actes ou les paroles d'autrui sont moralement condamnables parce qu'ils représentent une offense envers moi et me causent du tort. Les processus de type 1 interviennent sans que le sujet n'y prête nécessairement attention et ne demandent donc pas l'intervention de la mémoire de travail. Ils déclenchent des réponses automatiques à certains stimuli, correspondant donc à des opérations cognitives rapides, issues de l'évolution, de l'apprentissage implicite, ou de certains conditionnements (35). Ils vont par exemple être impliqués dans un jugement qui condamne moralement la relation consentie entre frères et sœurs. L'absence de justification sera corrélée à une absence d'utilisation de processus de type 2.

Une telle approche appliquée au domaine de la moralité permet d'envisager un continuum dans nos modes d'accès à la moralité, allant d'une construction du jugement moral très objective et raisonnée à une construction particulièrement intuitive et automatique. Elle se situe donc entre une approche strictement rationaliste et les approches sentimentalistes de la moralité. Selon Fine et Kennett, les personnes autistes seraient capables de délibération à propos de la morale, ce qui leur permettrait une moralité contrôlée et raisonnée. Mais elles auraient un manque de perception intuitive des raisons morales (18). On peut donc dire qu'elles utiliseraient peu ou pas les processus de type 1,

\footnotetext{
${ }^{6}$ L'article d'Evans et Stanovich présente une liste détaillée des caractéristiques des deux systèmes proposés par l'abondante littérature sur le sujet. Ils expliquent également le changement de terminologie de la notion de " système " à celle de "type ", la première impliquant à tort de concevoir deux systèmes singuliers et distincts qui sous-tendent exactement deux types de processus, alors que ces systèmes ou types sont en fait pluriels, impliquant différents processus cognitifs qui interagissent.
} 
mais presque exclusivement ceux de type 2, et se situeraient donc à l'extrémité du mode d'accès objectif aux raisons morales. Cette idée est compatible avec mon hypothèse, car elle expliquerait que les personnes autistes puissent être intransigeantes dans des cas où la moralité nécessite une transgression des règles morales, n'ayant pas une compréhension automatique de certaines raisons morales selon le contexte dans lequel elles s'inscrivent. Les personnes neurotypiques au contraire utiliseraient dans leurs évaluations morales des informations dont elles ne seraient pas conscientes de manière très rapide et inaccessible (34).

L'agentivité morale des personnes autistes découlerait donc d'un mode d'accès délibéré, objectif et non intuitif aux raisons morales. Celui-ci permet le développement d'une moralité en compensant la sous-utilisation des processus intuitifs ou des émotions. Les travaux de Brewer et collègues indiquent à ce propos qu'en ce qui concerne les jugements moraux, les personnes autistes auraient tendance à se baser plutôt sur des normes sociales apprises que sur l'information émotionnelle dont elles disposent (29). Cette moralité a des chances de s'avérer rigoriste et de donner lieu à des évaluations intransigeantes dans des cas où il faudrait considérer certaines circonstances atténuantes par exemple. Cela explique que certain-e-s autistes de hauts niveaux aient des troubles de capacité de "reason tracking» mais une forte "reason responsiveness" (18), c'est-à-dire qu'ils-elles sont capables de répondre aux raisons morales, de les appréhender, mais ont des difficultés à les détecter de manière automatique.

\section{Une surutilisation de processus de type 2 dans l'accès émotionnel}

Si les processus émotionnels n'entrent pas ou peu en jeu dans les évaluations morales des personnes autistes, la seconde partie de ce texte montrait qu'un certain ressenti pouvait néanmoins accompagner la compréhension des normes morales et qu'un certain besoin affectif pouvait être à l'origine de la construction d'une moralité, en tant qu'il motive à comprendre les normes qui régissent les domaines social et moral. En quoi les particularités socio-émotionnelles sous-tendent alors les différences au niveau des capacités morales chez les personnes autistes? S'agit-il d'un problème d'empathie comme c'est le cas chez les personnes dites psychopathes?

L'absence d'empathie caractéristique de la psychopathie a des conséquences sur leur moralité et pourrait même les exempter de responsabilité morale (31). Si les capacités empathiques et émotionnelles ont un rôle à jouer dans la construction de la moralité des neurotypiques, et qu'elles ont des incidences sur celle des personnes psychopathes, la compréhension des particularités émotionnelles des personnes autistes peut aussi éclairer celle de leurs particularités morales. Mais l'absence d'empathie chez les personnes autistes a été relativisée : certaines études montrent des particularités neuronales qui mettent en évidence des difficultés de reconnaissance émotionnelle (5) ou d'empathie "cognitive » (36), mais reconnaissent qu'il n'y a aucune différence avec les neurotypiques en ce qui concerne une autre forme d'empathie. Krahn et Fenton ont conceptualisé cette distinction et montré que les personnes autistes étaient pourvues d'empathie " affective » (37), c'est-à-dire qu'elles peuvent percevoir les réactions et émotions d'autrui et en faire elles-mêmes l'expérience. Ainsi, ce n'est pas une complète absence d'émotion ou d'empathie qui sous-tendrait leurs particularités morales. En effet, l'étude de Schneider et collègues ne montre aucune différence d'activation au niveau de l'amygdale droite entre les TSA et les groupes contrôles pendant les tâches de raisonnements moraux. Or, cette activation cérébrale serait impliquée dans les aspects empathiques basiques tels que la détection de la douleur (3). Cela corrobore la présence d'un " fort intérêt affectif » en ce qui a trait à la moralité (2). Le jeune homme autiste pourrait donc bien ressentir de la compassion pour la femme au supermarché, donc faire preuve d'empathie, mais son propre rapport avec cette compassion, issu de la détresse de cette femme, autrement dit son accès émotionnel, va s'avérer différent de celui des neurotypiques.

Les personnes autistes seraient sujettes à un déficit d' "identification et description " de "leurs propres émotions ॥ $(6,29)$, à des problèmes de régulation de leurs propres émotions $(7,8)$, et auraient 
un déficit de conscience (awareness) émotionnelle (9). Cela se retrouve au niveau hormonal et notamment au niveau de la libération d'ocytocine. Des études démontrent que des inhalations d'ocytocine favorisent la reconnaissance émotionnelle des personnes autistes (38). L'ensemble de ces déficits fait référence à des facultés qui concernent leur rapport à leurs propres expériences émotionnelles, autrement dit, les difficultés émotionnelles des personnes autistes seraient en lien avec leur accès émotionnel plutôt qu'avec le ressenti lui-même.

Les théories des processus duaux peuvent à nouveau éclairer notre compréhension de l'accès émotionnel. Que les émotions soient catégorisées comme appartenant à l'un ou l'autre des deux types de raisonnement, elles peuvent être accessibles à celui ou celle qui en fait l'expérience par l'un ou l'autre de ces types, donc grâce aux deux processus décrits par les théories des processus duaux : pour accéder à ma peur par exemple, je pourrai la comprendre et la reconnaitre soit de manière automatique, grâce à un ressenti subjectif intuitif (des processus de type 1), ou bien au contraire par des processus contrôlés et délibérés issus d'un raisonnement sur ce qu'est la peur en général (type 2). De la même façon, je pourrai me référer à ma compassion pour autrui de manière très automatique, par le caractère qualitatif - le « ce que cela fait » - d'être dans cet état de détresse, ou bien en raisonnant consciemment sur ce qui implique la détresse d'autrui et sur le fait de savoir si cette détresse qui mène à ma compassion justifie un jugement moral différent ou non selon les normes morales et sociales.

Les personnes autistes peuvent ressentir des émotions, mais leur compréhension ou connaissance de celles-ci - qui est à l'œuvre dans la construction de la moralité (30) - se fera alors plutôt par un processus délibéré et faisant intervenir le raisonnement, que par un accès intuitif, comme c'est le cas pour les neurotypiques. En effet, Losh et Capps analysent les discours d'enfants autistes et neurotypiques relatifs à leurs expériences émotionnelles et rapportent l'exemple d'un enfant neurotypique qui utilise des expériences subjectives passées pour décrire ce qu'est l'embarras, une émotion particulièrement impliquée dans des situations à caractère moral. Ils le comparent à celui d'un enfant autiste qui définit la honte comme ayant lieu " quand je fais quelque chose de mal et ensuite, je pourrais avoir des ennuis pour ça ». Selon les auteurs "cette description plutôt définitionnelle pourrait ne pas être dérivée de ses expériences personnelles " mais d' "une compréhension de la honte comme naissant d'interactions et de standards moraux » (39, traduction libre) soit d'éléments objectifs et extérieurs à la situation. II s'agit donc d'un accès non intuitif au ressenti qui correspond à des processus de type 2. Cela explique alors que leur "sensibilité morale » soit « structurée différemment » (2).

Ainsi, une personne autiste fera preuve d'empathie, mais l'émotion qu'elle ressentira alors fera l'objet d'un processus très raisonné pour être comprise, ce qui donnera lieu à une évaluation morale du même type. Le jeune homme qui condamne moralement l'action de voler peut compatir avec la détresse de cette femme, mais cette compassion sera évaluée comme étant distincte de la situation et des circonstances qui doivent servir à juger l'acte. Plutôt que de considérer que les personnes autistes sont dépourvues d'empathie et que cela affecte leurs capacités morales, il est possible de comprendre leurs particularités d'agentivité morale comme ayant pour origine des particularités d'accès à leurs propres émotions. Ces émotions sont bien issues d'une contagion émotionnelle, soit de leur empathie affective, mais elles feront l'objet d'une compréhension très objective et raisonnée, qui donnera lieu à des jugements moraux relativement inflexibles et rigoristes.

\section{Conclusion}

Lorsqu'on évalue une situation de transgression morale, différents jugements peuvent être formulés selon les personnes qui évaluent la situation. Elles s'avèrent plus ou moins flexibles ou rigoristes, plus ou moins attachées aux lois ou, au contraire, aux circonstances qui pourront atténuer la condamnation. Les jugements sont issus d'interactions entre le raisonnement, les intuitions, les émotions et les sentiments. 
La vie affective des personnes autistes est bien impliquée dans la construction de leur agentivité morale. Leurs émotions étant appréhendées par des processus contrôlés et basés sur des règles, elles donnent lieu à des jugements moraux souvent très rigoristes. Ainsi, les personnes autistes répondent aux critères des théories sentimentalistes et rationalistes de la moralité. Leur agentivité morale ne souffre pas de dissonance et la situation morale en question peut tout à fait avoir une tonalité émotionnelle. II a été montré dans ce texte que les personnes autistes utilisent plutôt des processus de type 2 dans la construction de leur agentivité morale et dans leur accès émotionnel, ce qui en fait des agents moraux particulièrement inflexibles, alors que pour les neurotypiques, les jugements moraux et l'accès émotionnel seront plus souvent sous-tendus par des processus intuitifs, heuristiques et inaccessibles - autrement dit, par des processus de type 1 - avant de devenir des jugements conscients (34).

Les personnes autistes, non seulement peuvent, mais ont aussi la volonté de comprendre la moralité et de s'y conformer. Le fait qu'elles s'intéressent à la moralité et qu'elles puissent construire certains systèmes pour s'y conformer le mieux possible me conforte à suivre l'opinion de Barnbaum selon laquelle une théorie morale adéquate doit pouvoir inclure les personnes autistes dans la communauté morale, leur reconnaissant des droits et devoirs (40) au même titre que les personnes neurotypiques. Ainsi, il ne s'agit pas seulement de reconnaitre des droits aux personnes autistes, mais elles doivent pouvoir être incluses dans la communauté morale en un sens étroit : leur connaissance et ressenti de la moralité une fois développés les rendent moralement responsables de leurs actes et autonomes en ce qui concerne leurs choix et décisions, comme c'est le cas pour les adultes neurotypiques. Le fonctionnement autistique de la moralité présente des particularités, expliquées dans cet article, mais celles-ci sont compatibles avec trois différents modèles de la moralité. Ainsi, les personnes autistes, pouvant être des agents moraux au même titre que les neurotypiques, ne devraient pas être exemptées de responsabilité ni mises sous la tutelle d'une autorité extérieure simplement en raison de leurs troubles autistiques.

II est envisageable que certaines personnes autistes ne puissent pas développer d'agentivité morale, notamment celles qui présentent d'importantes déficiences intellectuelles, ou d'autres troubles comorbides qui compromettent leur autonomie. Mais l'absence d'agentivité morale peut également se retrouver chez les personnes non autistes qui présenteraient une déficience intellectuelle ou dont l'autonomie serait compromise (dans le cas d'une démence sénile par exemple). Dans le futur, les recherches gagneraient à questionner le développement de l'agentivité morale dans d'autres psychopathologies ou conditions afin de spécifier les différentes caractéristiques que possèdent les individus qui constituent l'ensemble de la communauté morale. L'étude du développement de l'agentivité morale dans certaines psychopathologies pourrait également permettre d'enrichir et de préciser les différents processus cognitifs qui sous-tendent un tel développement. La notion de communauté morale pourrait alors être mieux définie grâce à une description fine de différents types d'agentivité morale. Toutes ces clarifications pourraient alors avoir des répercussions sur nos différentes conceptions de la moralité et éventuellement mener à de nouvelles conclusions à propos de la responsabilité légale des individus.

\section{Références}

1. American Psychiatric Association. Diagnostic and statistical manual of mental disorders: DSM5. Washington, D.C.: American Psychiatric Association; 2013.

2. McGeer V. Varieties of moral agency: Lessons from autism (and psychopathy). In: Moral Psychology: The Neuroscience of Morality: Emotion, Brain Disorders, and Development, W Sinnott-Armstrong, ed. (Vol. 3), Cambridge MA: MIT Press, 2007. P. 227-258.

3. Schneider K, Pauly KD, Gossen A, Mevissen L, Michel TM, Gur RC, et al. Neural correlates of moral reasoning in autism spectrum disorder. Soc Cogn Affect Neurocsi. 2012;nss051.

4. Shulman C, Guberman A, Shiling N, Bauminger N. Moral and social reasoning in autism spectrum disorders. J Autism Dev Disord. 2012;42(7):1364-76. 
5. Michel TM, Herholz S, Finkelmeier A, Schneider F, Brügmann E, Haeck M, et al. The neuronal correlates of empathy in autism spectrum disorders. Eur Psychiatry. 2011;26:1467.

6. Hill E, Berthoz S, Frith U. Brief report: cognitive processing of own emotions in individuals with autistic spectrum disorder and in their relatives. J Autism Dev Disord. avr 2004;34(2):229-35.

7. Glaser SE, Shaw SR. Emotion regulation and development in children with autism and $22 q 13$ deletion syndrome: Evidence for group differences. Res Autism Spectr Disord. 2011; 5(2):926934

8. Samson AC, Huber O, Gross JJ. Emotion regulation in Asperger's syndrome and highfunctioning autism. Emotion. 2012;12(4):659-65.

9. Silani G, Bird G, Brindley R, Singer T, Frith C, Frith U. Levels of emotional awareness and autism: an fMRI study. Soc Neurosci. 2008;3(2):97-112.

10. Kennett J. Autism, empathy and moral agency. Philosophical Quarterly. 2002; 52(208):340357.

11. Decety J, Cowell JM. The complex relation between morality and empathy. Trends Cogn Sci. juill 2014;18(7):337-9.

12. Carruthers $P$. Autism as mindblindness: an elaboration and partial defence. In: Carruthers $P$, Smith PK, éditeurs. Theories of Theories of Mind. Cambridge University Press; 1996. p. 25776.

13. Lombardo MV, Barnes JL, Wheelwright SJ, Baron-Cohen S. Self-referential cognition and empathy in autism. PLoS ONE. 2007;2(9):e883.

14. Greimel E, Schulte-Rüther M, Kircher T, Kamp-Becker I, Remschmidt H, Fink GR, et al. Neural mechanisms of empathy in adolescents with autism spectrum disorder and their fathers. Neurolmage. 2010;49(1):1055-1065.

15. lacoboni M, Dapretto $M$. The mirror neuron system and the consequences of its dysfunction. Nat Rev Neurosci. 2006;7(12):942-51.

16. Rizzolatti G, Fabbri-Destro M. Mirror neurons: from discovery to autism. Exp Brain Res Exp Hirnforsch Expérimentation Cérébrale. 2010;200:223-237.

17. Benn P. Freedom, resentment, and the psychopath. Philos Psychiatry Psychol. 1999;6(1):2939.

18. Kennett J, Fine C. Will the real moral judgment please stand up? Ethic Theory Moral Pr Ethical Theory Moral Pract. 2009;12(1):77-96.

19. Blair RJR. Brief report: Morality in the autistic child. J Autism Dev Disord. 1996;26(5):571-9.

20. Grandin T. Thinking in Pictures: And Other Reports from My Life with Autism. New York: Doubleday; 1995.

21. Kohlberg L. Child Psychology and Childhood Education: A Cognitive-Developmental View. New York: Longman; 1987.

22. Krebs DL, Denton K. Toward a more pragmatic approach to morality: a critical evaluation of Kohlberg's model. Psychol Rev. 2005;112(3):629.

23. Leslie A Mallon, Ron, Dicorcia, Jennifer, Mallon R, Dicorcia J. Transgressors, victims, and cry babies: Is basic moral judgment spared in autism? Soc Neurosci. 2006;1(3-4):270-283.

24. De Vignemont F, Frith U. Autism, morality and empathy. In: Moral Psychology: The Neuroscience of Morality: Emotion, Brain Disorders, and Development, W Sinnott-Armstrong, ed. (Vol. 3), Cambridge MA: MIT Press, 2007;273-80.

25. Jones EJH, Webb SJ, Estes A, Dawson G. Rule learning in autism: the role of reward type and social context. Dev Neuropsychol. 2013;38(1):58-77.

26. Keel JH. A Study of Moral Reasoning in Adults with Autism. University of North Carolina at Chapel Hill; 1993.

27. Bolling DZ, Pitskel NB, Deen B, Crowley MJ, McPartland JC, Kaiser MD, et al. Enhanced neural responses to rule violation in children with autism: a comparison to social exclusion. Dev Cogn Neurosci. 2011;1(3):280-94.

28. Faucher L, Arnaud S. Raisons morales, motivations morales et psychopathologies. Sous presse. 
29. Brewer R, Marsh AA, Catmur C, Cardinale EM, Stoycos S, Cook R, et al. The impact of autism spectrum disorder and alexithymia on judgments of moral acceptability. J Abnorm Psychol. 2015;124(3):589.

30. Haidt J. The emotional dog and its rational tail: a social intuitionist approach to moral judgment. Psychol Rev. 2001;108(4):814-834.

31. Levy N. The responsibility of the Ppychopath revisited. Philos Psychiatry Psychol. 2007;14(2):129-138.

32. Prinz JJ. Against empathy. SJP South J Philos. 2011;49:214-33.

33. Berg JM, Smith SF, Watts AL, Ammirati R, Green SE, Lilienfeld SO. Misconceptions regarding psychopathic personality: implications for clinical practice and research. Neuropsychiatry. 2013;3(1):63-74.

34. Dubljević V, Racine E. The ADC of moral judgment: Opening the black box of moral intuitions with heuristics about agents, deeds, and consequences. AJOB Neurosci. 2014;5(4):3-20.

35. Evans JSB, Stanovich KE. Dual-process theories of higher cognition advancing the debate. Perspect Psychol Sci. 2013;8(3):223-41.

36. Schwenck C, Mergenthaler J, Keller K, Zech J, Salehi S, Taurines R, et al. Empathy in children with autism and conduct disorder: Group-specific profiles and developmental aspects. J Child Psychol Psychiatry. 2012;53(6):651-9.

37. Krahn T, Fenton A. Autism, empathy and questions of moral agency. J Theory Soc Behav. 2009;39(2):145-66.

38. Guastella AJ, Einfeld SL, Gray KM, Rinehart NJ, Tonge BJ, Lambert TJ, et al. Intranasal oxytocin improves emotion recognition for youth with Autism Spectrum Disorders. Biol Psychiatry. 1 avr 2010;67(7):692-4.

39. Losh M, Capps L. Understanding of emotional experience in autism: Insights from the personal accounts of high-functioning children with autism. Dev Psychol. 2006;42(5):809.

40. Barnbaum DR. The Ethics of Autism: Among Them, But Not of Them. Bloomington: Indiana University Press; 2008. 